\title{
AN EQUIVARIANT TIETZE EXTENSION THEOREM FOR PROPER ACTIONS OF LOCALLY COMPACT GROUPS
}

\author{
AASA FERAGEN
}

\begin{abstract}
The classical Tietze extension theorem asserts that any continuous map $f: A \rightarrow \mathbb{R}^{n}$ from a closed subset $A$ of a normal space $X$ admits a continuous extension $F: X \rightarrow \mathbb{R}^{n}$. The Tietze-Gleason theorem is an equivariant version of the same theorem for spaces with actions of compact groups, proved by A. Gleason in the 1950s. Here we prove the following version of the theorem for proper actions of locally compact groups: let $G$ be a locally compact group acting properly on a completely normal space $X$ such that $X / G$ is paracompact, and let $A$ be a closed $G$-invariant subset of $X$. Suppose $\rho: G \rightarrow G L(n, \mathbb{R})$ is a representation; now any continuous $G$-equivariant mep $f: A \rightarrow \mathbb{R}^{n}(\rho)$ admits a continuous $G$-equivariant extension $F: X \rightarrow \mathbb{R}^{n}(\rho)$.
\end{abstract}

\section{INTRODUCTION}

The Tietze extension theorem is one of the most basic, and perhaps the most wellknown, continuous extension theorems. An equivariant version of it for compact groups was proven by A. Gleason in the 1950s [G], using the Haar integral to "average" over the group. The same technique does not work for non-compact groups since the Haar integral does not generally converge over non-compact groups. However, for proper actions of locally compact groups, we can use slices to pass from a solution for a compact subgroup on a slice, to a solution for the whole group on the corresponding tubular neighborhood.

\section{Prerequisites}

Let $G$ be a locally compact Hausdorff topological group, and let $X$ be a topological space. An action of $G$ on $X$ is a continuous map $\Phi: G \times X \rightarrow X$ such that $\Phi(e, x)=x$ for all $x \in X$, where $e$ is the neutral element of the group, and such that $\Phi\left(g^{\prime}, \Phi(g, x)\right)=\Phi\left(g^{\prime} g, x\right)$. We usually denote $\Phi(g, x)=g x$.

Two subsets $A$ and $B$ of a topological space $X$ are said to be separated in case $\bar{A} \cap B=\emptyset=A \cap \bar{B}$. A topological space $X$ is completely normal if any two separated subsets $A$ and $B$ of $X$ can be separated by disjoint neighborhoods $V_{A}$ and $V_{B}$. A topological space $X$ is completely normal if and only if any subspace of $X$ is normal [?, Theorem II.5.1].

The action of $G$ on a completely regular space $X$ is a Cartan action if, for any $x \in X$, there exists a neighborhood $V$ of $x$ in $X$ such that $\{g \in G \mid g V \cap V \neq \emptyset\}$ is relatively compact in $G$. The action is proper if for any pair of points $x, y \in X$ we can find neighborhoods $V_{x}$ and $V_{y}$ of $x$ and $y$ respectively, such that the set $\left\{g \in G \mid g V_{x} \cap V_{y} \neq \emptyset\right\}$ is relatively compact in $G$. Even more restrictively, we call the action Palais proper if for each $x \in X$ we can find a neighborhood $V_{x}$ of $x$ in $X$ such that for any other point $y \in X$ we can find a neighborhood $V_{y}$ of $y$ in $X$ such that the set $\left\{g \in G \mid g V_{x} \cap V_{y} \neq \emptyset\right\}$ is relatively compact in $G$.

2000 Mathematics Subject Classification. 57S20.

Key words and phrases. Proper actions, equivariant extension, Tietze.

The research leading to this article was financed by Helsingin Yliopiston Tiedesti. 
It is clear that any Palais proper action is proper, and that any proper action is a Cartan action.

Let $H$ be a closed subgroup of $G$. A subset $S$ of a $G$-space $X$ is an $H$-slice if $G S$ is open in $X$ and there exists a $G$-map $f: G S \rightarrow G / H$ such that $S=f^{-1}(e H)$.

Lemma 1. Suppose that $S \subset X$ is an $H$-slice. Then

i) $g S \cap S \neq \emptyset$ implies that $g \in H$,

ii) $S$ is closed.

Proof. i) Suppose that $g S \cap S \neq \emptyset$, and let $f: G S \rightarrow G / H$ be such that $S=f^{-1}(e H)$. Then for some $s \in S$, also $g s \in S$ and thus $g H=g(e H)=$ $g f(s)=f(g s) \in f(S)=e H$. But that implies $g \in H$.

ii) This is trivial since $f$ is continuous and $e H$ is closed (it is a point in $G / H$, which is Hausdorff because $H$ is compact).

Given a closed subgroup $H$ of $G$ and an $H$-space $S$ there is an action of $H$ on the product $G \times S$ given by $h(g, s)=\left(g h^{-1}, h s\right)$. We denote by $G \times{ }_{H} S$ the quotient space $(G \times S) / H$, which is called the twisted product of $G$ and $S$ with respect to $H$. There is an action of $G$ on $G \times_{H} S$ defined by the formula $\bar{g}[g, s]=[\bar{g} g, s]$.

Proposition 2. Let $H$ be a closed subgroup of $G$ and let $S$ be an $H$-invariant subset of $X$. Then $S$ is an $H$-slice if and only if $G S$ is an open subset of $X$ and

$$
G \times_{H} S \approx_{G} G S
$$

We say that the open set $G S$ is a tubular neighborhood (of $x$ ) if $S$ is an $H$-slice (a slice at $x$ ). A tubular covering of a $G$-space $X$ is a covering of $X$ by tubular neighborhoods.

\section{The Equivariant Tietze EXtension theOREM}

Theorem 3. Let $G$ be a locally compact group and let $X$ be a completely normal proper $G$-space such that $X / G$ is paracompact. Let $A \subset X$ be a closed $G$-subset. Let $\rho: G \rightarrow \operatorname{GL}(n, \mathbb{R})$ be a representation and let $\varphi: A \rightarrow \mathbb{R}^{n}(\rho)$ be a $G$-map. Then there exists a continuous $G$-extension $\psi: X \rightarrow \mathbb{R}^{n}(\rho)$.

By [B, Theorem 2.5], any Cartan $G$-space $X$ has a tubular covering $\left\{G S_{i}\right\}_{i \in I}$ where each $S_{i}$ is an $H_{i}$-slice for some compact subgroup $H_{i}$ of $G$.

Lemma 4. Let $G$ be a locally compact group, let $X$ and $Y$ be $G$-spaces, let $S$ be a $H$-slice for a compact subgroup $H$ of $G$, and let $V$ be an $H$-invariant subset of $S$. Let $f: V \rightarrow Y$ be an $H$-map. Then there exists a unique continuous $G$-map $\tilde{f}: G V \rightarrow Y$ which extends $f$.

Proof. The only possible $G$-function $G V \rightarrow Y$ which extends $f$ is $\tilde{f}$ defined by

$$
\tilde{f}(g v)=g f(v) \forall g \in G, v \in V .
$$

We show that the function $\tilde{f}$ is well-defined. If $g v=g^{\prime} v^{\prime}$ for some $g, g^{\prime} \in G$ and $v, v^{\prime} \in V \subset S$ then $v=g^{-1} g^{\prime} v^{\prime}$ implying $S \cap\left(g^{-1} g^{\prime}\right) S \neq \emptyset$. Since $S$ is a slice, we get by Lemma 1 that $g^{-1} g^{\prime} \in H$. Since $f$ is an $H$-map, then $f(v)=f\left(g^{-1} g^{\prime} v^{\prime}\right)=$ $g^{-1} g^{\prime} f\left(v^{\prime}\right)$ giving $g f(v)=g^{\prime} f\left(v^{\prime}\right)$. Hence $\tilde{f}(g v)=\tilde{f}\left(g^{\prime} v^{\prime}\right)$ and $\tilde{f}$ is well defined.

Equivariance is clear from the definition, and $\tilde{f}$ is continuous since the diagram below commutes and the left vertical projection is a quotient map because it is the projection $G \times V \rightarrow(G \times V) / H$ to the orbit space of a compact group action. 


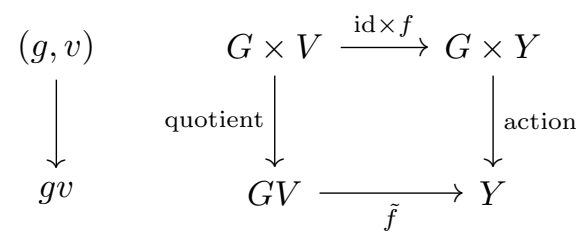

Corollary 5. Let $i \in I$, and denote by $A_{i}=A \cap G S_{i}$. The map $f_{i}=f \mid: A_{i} \rightarrow$ $\mathbb{R}^{n}(\rho)$ admits a $G$-equivariant continuous extension $F_{i}: G S_{i} \rightarrow \mathbb{R}^{n}(\rho)$.

Proof. The map

$$
\tilde{f}_{i}: A_{i} \cap S_{i} \rightarrow \mathbb{R}^{n}(\rho \mid)
$$

where $\rho \mid: H_{i} \rightarrow G L(n, \mathbb{R})$, admits an $H_{i}$-equivariant extension

$$
\tilde{F}_{i}: S_{i} \rightarrow \mathbb{R}^{n}(\rho \mid)
$$

by [G]. By Lemma 4 there exists a $G$-equivariant continuous extension

$$
F_{i}: G S_{i} \rightarrow \mathbb{R}^{n}(\rho)
$$

given by $F_{i}(g s)=g \tilde{F}_{i}(s)$, and we easily see that $F_{i} \mid A_{i}=f_{i}$.

Lemma 6. Suppose that $\left\{U_{i}\right\}_{i \in I}$ is a locally finite covering of a topological space $X$. Then $\left\{\bar{U}_{i}\right\}_{i \in I}$ is locally finite as well.

Proof. Suppose $x \in X$; then there exists a neighborhood $U$ of $x$ which meets only finitely many $U_{i}$. But if $U \cap U_{j}=\emptyset$, then $U \subset U_{j}^{C}$ and then since $U$ is open, $U \subset \operatorname{int}\left(U_{j}^{C}\right)=\left(\bar{U}_{j}\right)^{C}$, so $U \cap \bar{U}_{j}=\emptyset$. Hence $U$ meets $\bar{U}_{i}$ only for those $i \in I$ for which $U$ meets $U_{i}$, and there are only finitely many such $i \in I$.

In particular, the covering $\left\{\overline{G S}_{i}\right\}_{i \in I}$ is locally finite.

Since $X / G$ is paracompact, the covering $\left\{G S_{i}\right\}_{i \in I}$ has a locally finite open $G$-refinement $\left\{U_{j}^{\prime}\right\}_{j \in J}$. Because Hausdorff paracompact spaces are normal and $\left\{U_{j}^{\prime}\right\}_{j \in J}$ is, in particular, point-finite, we may find a $G$-refinement $\left\{U_{j}\right\}_{j \in J}$ of $\left\{U_{j}^{\prime}\right\}_{j \in J}$ such that $\bar{U}_{j} \subset U_{j}^{\prime}$ for all $j \in J .\left(\bar{U}_{j}=\operatorname{cl}_{X} U_{j}\right.$. $)$ By Lemma 6, there exists a continuous $G$-equivariant extension $F_{i}: G S_{i} \rightarrow \mathbb{R}^{n}(\rho)$ of $f_{i}$.

Well-order the index set $I$, and let $i \in I$. Suppose that for all $j<i$ we have extensions $F_{j}: A_{j} \rightarrow Y$ of $f \mid A \cap \bar{U}_{j}$, where $A_{j}=A \cup \bar{V}_{j}$ and where $\bar{V}_{j}$ is the closure in $\bar{U}_{j}$ of a neighborhood $V_{j}$ of $A \cap \bar{U}_{j}$ in $\bar{U}_{j}$, such that all $F_{j}$ agree on their common area of definition. We claim that there exists a neighborhood $V_{i}$ of $A \cap \bar{U}_{i}$ in $\bar{U}_{i}$ and an extension $F_{i}: A_{i} \rightarrow Y$ of $f \mid A \cap \bar{U}_{i}$ where $A_{i}=A \cup \bar{V}_{i}\left(\bar{V}_{i}=\mathrm{cl}_{\bar{U}_{i}} V_{i}\right)$, which agrees with every $F_{j}, j<i$, on their common area of definition.

The functions $F_{j}$ where $j<i$ combine to a function $\overline{F_{i}}: \bigcup_{j<i} A_{j} \rightarrow Y$. This function $\bar{F}_{i}$ is continuous by the local finiteness of the family $\left\{\bar{V}_{j}\right\}_{j<i} \cup\{A\}$ (since $\bigcup_{j<i} A_{j}=A \cup \bigcup_{j<i} \bar{V}_{j}$ ). Furthermore, $\bigcup_{j<i} A_{j}$ is closed, again by the local finiteness of the family $\left\{\bar{V}_{j}\right\}_{j<i} \cup\{A\}$.

Now by Lemma 6 the map $\bar{F}_{i} \mid\left(\bar{U}_{i} \cap\left(\bigcup_{j<i} A_{j}\right)\right)$ has a $G$-extension $\tilde{F}: \bar{V}_{i} \rightarrow Y$ where $V_{i}$ is a neighborhood of $\bar{U}_{i} \cap\left(\bigcup_{j<i} A_{j}\right)$ in $\bar{U}_{i}$. In particular $V_{i}$ is a neighborhood of $A \cap \bar{U}_{i}$ in $\bar{U}_{i}$. Now we use this to obtain a map $F_{i}: A_{i} \rightarrow Y$ where $A_{i}=A \cup \bar{V}_{i}$, by setting:

$$
\begin{aligned}
& F_{i}\left|A=\bar{F}_{i}\right| A=f \mid A \\
& F_{i} \mid \bar{V}_{i}=\tilde{F}
\end{aligned}
$$

Obviously $F_{i}$ agrees with each $F_{j}(j<i)$ on their common area of definition. 
Hence by transfinite construction there exists, for every $i \in I$, a neighborhood $V_{i}$ of $A \cap \bar{U}_{i}$ in $\bar{U}_{i}$ and an extension $F_{i}: A_{i} \rightarrow Y$ of $f \mid A \cap \bar{U}_{i}$ where $A_{i}=A \cup \bar{V}_{i}$ such that $F_{i}$ agrees with any $F_{j}$ on their common area of definition.

Using the above we obtain a map $F: \bar{W} \rightarrow Y$ where $W=\bigcup_{i \in I} V_{i} \cap U_{i}$ by setting

$$
F(x)=F_{i}(x) \text { when } x \in \bar{V}_{i} .
$$

The map is well-defined; it is continuous by local finiteness and closedness of the $\bar{V}_{i}$, and $W$ is a neighborhood of $A$ since

$$
A \subset \bigcup_{i \in I}\left(A \cap U_{i}\right) \subset \bigcup_{i \in I}\left(V_{i} \cap U_{i}\right)=W
$$

\section{REFERENCES}

[B] H. Biller, Characterizations of proper actions, Math. Proc. Camb. Phil. Soc. (2004), 136, 429-439.

[G] A. Gleason, Spaces with a compact Lie group of transformations, Proc. Amer. Math. Soc, 1 (1950), 35-43. 